\title{
ROLE OF LABORATORY SERVICES AS SUPPORT OF HEALTH CARE
}

\author{
Naima Muazzam
}

Health care means both preventive and curative measures. In both the methods, the laboratory services play a vital role to help the clinicians.

It is now universally accepted that "Prevention is better than cure". but in many developing countries including Bangladesh, adequate emphasis has not yet been given on the preventive aspects of Health care. As a result many preventable diseases are still serious health problem in our country.

In the preventive field certain laboratory tests can help the clinicians to a great extent :

(a) Vaccination against some infectious diseases are normal preventive measures. In this field certain preliminary laboratory tests are essential before giving the vaccine.As for example. Mantoux test before BCG Vaccination and certain serological tests before Hepatitis B Virus vaccination.

(b) Certain diseases like Malaria and Kala-azar, which are also preventable, still remain as important health hazards in some developing countries like Bangladesh. Preventive measures play the main role in the eradication of these diseases.

To explain this point, we are to go a little deep into the disease processes. For example, if we consider kala-azar, we know that it is a chronic debilitating disease. But, in the beginning, the patient may remain unaware of the disease and continue to be ambulent with some fever only. However, when full-blown kalaazar develops, then it be- comes easy for the clinicians to diagnose and treat the cases.

Besides, in an endermic zone for kala-azar a number of persons may remain asymptomatic carriers of leishmania. So, for the prevention and eradication of kala-azar, such carriers and asymptomaic patients should be detected and treated, Otherwise, they will act as reseruoir for the spread of Kala-azar.To detect such cases, certain laboratory tests like the Enzyme Linked Immunoabsorbent Assay (ELISA), Direct agglutination test (DAT) and Indirect Fluorescent Antibody Test (IFAT) are available ${ }^{1}$.

(c) Before Blood transfusion the donors should undergo a number of laboratory tests to detect certain diseases, so that such diseases are not transferred to the recepients. In this way spread of diseases like Hepatitis B and AIDS can be prevented or reduced to a great extent.

(d) Even before performing a simple oparation like circumcision. bleeding and clotting times (BT. CT) should be determined to prevent untoward incidents in cases of haemophila and bleeding diathesis.

Dr.Naima Muazzam MBBS.M. Phil(Micro). Assoc.Prof of Microbiologiy. Dhaka Medical college. 
Thus, laboratory services can play an important role in prevention of many preventable diseases.

In the curative field, laboratory tests are often essential for correct diagonosis. treatment and follow-up of the cases:

(a) Patients come to the clinicians with certain complaints and the clinicians take history, examine the patient and note down the signs and symptoms and then they come to a probable diagonosis. As some signs and symptoms are often common to more than one disease, the clinicians may need some laboratory investigations to exclude some possibilities and to establish the correct diagnosis. In the absence of reliable laboratory services, the suffering of the patient may be prolonged or even the diagnosis may be worng. The following examples are noteworthy:

(i) A patient with fever may be a problem for the clinician as several distinct diseases may have the same symptom of pyrexia. A simple blood count may point out if it is a case of pyogenic infections like acute appendicitis, acute sulpingitis or some other infections like lobar pneumonia or even septicaemia. The findings of blood count alongwith the signs and symptoms may indicate the correct diagnosis.

While examining the blood film if malarial parasite is found, then the diagnosis is complete. Besides, a low blood count or leucopenia may indicate other conditions for which the clinicians may ask for some other serlogical investigations like Widal, VDRL, HBS antigen etc. depending on the findings of the clinical examinitions. Thus proper laboratory investigations may go a long way to improve the health care system.

(ii) Gastro-intestional trouble is another common presenting symptom in our country. A routine stool analysis is helpful for the quick diagnosis of intestinal helminthiasis, bacterial or parasitic infection of the-G.I. tract. For further information, culture and sensitivity test may also be done.

(iii) For confirmation of diabetes mellitus, besides glucose tolerance test (GTT), random blood sugar estimation and urine examination are also necessary.

Routine urine analysis and even culture sensitivity test will help to diagnose the urinary tract infection and kidney diseases.

Now, let us see how far laboratory service is available in our country and what are the problems in getting satisfactory laboratory services.

In our country the laboratory services are not well supervised by the Health Depertment. It must be ensured that qualified pathologists, microbiologists, biochemists and technicians are employed for the purpose.

Another practical problem is that the clinicians often do not supply the relevent clinical data with the requisition slips. This ia essential to ensure proper investigatons. The clinical datas will provide the necessary informations about the type of findings in which the clinicians want definite answer, so that correct diagnosis may be made.

For example in case of Widal test, signs, symptomps and provisional diagnosis are necessory for proper interpretation of the results of the test. Without the above 
mentioned informations, the pathologists or laboratory personnels can not give proper interpretation or comment on the results of the Widal test.

In certain investigations, the age of the patient and the type of treatment already given are also helpful.

The last,but not the least is that, a close contact between the clinicians and the laboratory specialists are needed for the best service to the patients.If a finding is not compatible with the clinical diagnosis or if the result of the investigation is not clear to the clinicians, they should directly contact the laboratory personnel and discuss the matter to avoid loss of time.

It is unfortunate that academic autopsy is almost absent in our country. As a result, there is no improvement in the medical science and research. After careful diagnosis and treatment if a patient dies, an autopsy will help to find if the diagnosis was correct. If found correct, the confidence of the clinicians will be enhanced. If it is found that the diagnosis and treatment were wrong, then the autopsy findings will help clinicians to improve their knowledge for managing similar cases in future ${ }^{2}$.

Because of regular clinico-pathological conference on difficult cases and regular academic autopsy of difficult or undiagnosed cases, the laboratory specialists serve as the 'consultants of the Consultants' in the Advanced countries for the benefit of the patient. The purpose of autopsy is to improve the knowlege of both the cliniclans and the laboratory personnels.

Let us hope that better laboratory services are available and academic autopsy is encouraged in our country for improving our knowledge of medical science and providing improved services and treatment to the patients.

\section{References}

1. Muazzam, N., Rahman, KM., Mia, RA and Asna, SMZH; Indirect Fluorescent Antibody Test in the Serodignosis of Vlsceral Leishmaniasis in Bangladesh, BD. Med. Res. council Bull. 18 (2), 77-81 p.,1992

2. Muazzam, M.G., Khaleque K. A, and Ibrahim, M: Hepatic Ascariasis, J. Trop. Med. \& Hgg. $63: 95-97,1960$ 